\title{
Incidencia de enterocolitis necrosante en niños prematuros alimentados precozmente
}

\author{
Luis Alfonso Pérez ${ }^{1,2}$, Germán Lauro Pradilla ${ }^{3,4}$, Gaby Díaz ${ }^{5}$, Sandra María Bayter ${ }^{6}$ \\ ${ }^{1}$ Departamento de Pediatría, Facultad de Salud, Escuela de Medicina, Universidad Industrial de Santander, \\ Bucaramanga, Colombia \\ 2 Unidad Neonatal, Hospital Universitario de Santander, Bucaramanga, Colombia \\ 3 Departamento de Pediatría, Facultad de Salud, Escuela de Medicina, Universidad Industrial de Santander, \\ Bucaramanga, Colombia \\ ${ }^{4}$ Servicio de Urgencias, Hospital Universitario de Santander, Bucaramanga, Colombia \\ 5 Unidad de Cuidado Intensivo Cardiovascular Pediátrico, Fundación Cardiovascular de Colombia, Bucaramanga, \\ Colombia \\ 6 Salud Total EPS, Bucaramanga, Colombia
}

Introducción. La enterocolitis necrosante es una catástrofe multifactorial de los recién nacidos, generada por vasoconstricción visceral. Se discute el papel que juega el momento, tipo y velocidad de los incrementos de la vía oral en la patogenia de la entidad.

Objetivo. Comparar la incidencia de enterocolitis necrosante y la mortalidad entre prematuros en quienes se inició alimentación entérica a las 48 horas de vida extrauterina, frente a otros en quienes se empezó al quinto día de vida.

Materiales y métodos. Se llevó a cabo un ensayo clínico controlado de 239 neonatos de 750 a 1.500 gramos de peso y de 27 a 32 semanas de edad de gestación, asignados al azar, 135 a alimentación temprana y 104 a alimentación tardía con leche materna o fórmula láctea para prematuros. Se inició con un mililitro cada seis horas, para progresar en intervalos de tres horas y un incremento diario de hasta $20 \mathrm{ml} / \mathrm{kg}$ hasta llegar a $150 \mathrm{ml} / \mathrm{kg}$ al día, siempre y cuando no se observaran signos o síntomas de intolerancia a la vía oral.

Resultados. Se presentaron 14 casos de enterocolitis necrosante en el grupo de alimentación temprana (10,4\%) y nueve $(8,7 \%)$ en el de alimentación tardía ( $\left.R R=1,22 ; I_{95} 0,49-3,20 ; p=0,652\right)$. Cinco $(3,7 \%)$ niños fallecieron en el grupo de alimentación temprana $\left(\mathrm{IC}_{95} \% 1,4-8,9\right)$ y ocho $(7,7 \%)$, en el de alimentación tardía $\left(\mathrm{IC}_{95} \% 3,6-15,0\right)$, sin que estas diferencias fueran significativas ( $R R=0,46$; $\left.\mathrm{IC}_{95 \%} 0,12-1,60 ; \mathrm{p}=0,182\right)$.

Conclusión. El iniciar en forma temprana la alimentación entérica en neonatos prematuros no aumenta el riesgo de enterocolitis necrosante ni el de mortalidad y sí representa grandes ventajas para estos pacientes.

Palabras clave: enterocolitis necrosante, alimentación entérica, leche humana, recién nacido.

\section{Necrotizing enterocolitis among preterm newborns with early feeding}

Introduction. The development of necrotizing enterocolitis is a common and serious risk to newborns. It is caused by splanchnic bed vasoconstriction that can produce intestinal necrosis. The role of onset time, type and, speed enhancements of The factors of enteral feeding related with necrotizing enterocolitis genesis such as feeding onset, type and frequency are not well understood.

Objective. The incidence of necrotizing enterocolitis and mortality among infants will be compared in infants whose enteral feeding was initiated 48 hours after birth to those whose feeding began on day 5 postpartum.

Materials and methods. A controlled clinical trial was conducted among 239 newborns weighing between $750-1,500 \mathrm{~g}$ and a gestation age of 27-32 weeks of gestational age. The infants were randomly assigned to two groups--135 to an early feeding regime and 104 to delayed feeding. Breast milk or formula milk was used. Feeding was begun with one $\mathrm{ml}$ every six hours, progressing to three hour intervals and $20 \mathrm{ml} / \mathrm{kg}$ daily. This was increased to $150 \mathrm{ml} / \mathrm{kg}$-d if the infant condition remained stable and it manifested no oral intolerance.

\footnotetext{
Contribuciones de los autores:

Luis Alfonso Pérez: diseño, captación de pacientes, análisis y elaboración del manuscrito.

Germán Lauro Pradilla: captación de pacientes y elaboración del manuscrito.

Gaby Díaz: diseño, captación de pacientes, análisis y elaboración del manuscrito.

Sandra María Bayter: diseño, captación de pacientes, análisis y elaboración del manuscrito.
} 
Results. In the early feeding group, 14 (10;4 \%) necrotizing enterocolitis cases occurred, and in the late feeding group, $9(8.7 \%)$ occurred $(\mathrm{RR}=1.22,95 \% \mathrm{Cl} 0.49-3.20, p=0.65)$. Five children died in early feeding group $(3.7 \%, 95 \% \mathrm{Cl} 1.4-8.9)$ and eight in late feeding group $(7.7 \%, 95 \% \mathrm{Cl} 3.6-15.0, \mathrm{RR}=$ $0.46,95 \% \mathrm{Cl} 0.12-1,60, p=0.18)$.

Conclusion. Early enteral feeding in preterm newborns does not increase the risk of necrotizing enterocolitis or mortality among them; however it represents nutritional advantages for these infants.

Key words: Enterocolitis, necrotizing; enteral nutrition; milk, human; infant, newborn.

La enterocolitis necrosante es un situación abdominal catastrófica en el recién nacido (1); es más frecuente en el niño prematuro (90\%) (2-5) y su origen es multifactorial. Entre los factores de riesgo propuestos están: la prematuridad, que es el más claramente demostrado (3-5); la asfixia al nacer, que genera redistribución vascular y restricción del crecimiento intrauterino $(2,6)$, y la alimentación entérica $(7,8)$. Todos desencadenan un proceso inflamatorio que lleva a la vasoconstricción del lecho visceral y provoca necrosis (9-11).

La alimentación del recién nacido prematuro suscita controversias. Los esquemas de alimentación entérica en el recién nacido prematuro van dirigidos a disminuir la frecuencia de enterocolitis necrosante. Durante un tiempo se promulgó que el postergar el inicio de la alimentación prevenía la aparición de enterocolitis necrosante $(12,13)$. Sin embargo, se ha demostrado que la alimentación entérica mínima, ofrecida tempranamente, tiene ventajas como generar estímulo trófico, aumentar la producción enzimática y mejorar la motilidad intestinal $(14,15)$. Además, algunos estudios han encontrado que el inicio temprano de la vía oral no aumenta el riesgo de enterocolitis $(16,17)$. Igualmente, se discute el papel que podría jugar la velocidad en los incrementos de la vía oral (18), aunque algunos estudios no encuentran relación alguna $(19,20)$.

Por lo anterior, el objetivo principal de este trabajo fue comparar la incidencia de enterocolitis necrosante y la mortalidad en un grupo de niños en quienes se inició la alimentación entérica a las 48 horas de vida extrauterina y otro en que se hizo al quinto día.

\section{Materiales y métodos}

Entre agosto de 1997 y enero de 2005, se llevó a cabo un ensayo clínico controlado que incluyó niños menores de dos días de vida que estuviesen

\section{Correspondencia:}

Luis Alfonso Pérez, Carrera 30 № 53-23, apartamento 1002, Edificio Vizcaya Bolarqui, Bucaramanga, Colombia

Teléfono: (57) 634 6110, extensión 329; fax: (57) 6433014

Iperezvera@hotmail.com

Recibido:17/01/11; aceptado:30/01/11 hospitalizados en las salas de recién nacidos del Hospital Universitario Ramón González Valencia de Bucaramanga, Colombia. Los padres o representantes legales fueron informados de la investigación y dieron su consentimiento informado por escrito. El trabajo fue aprobado por el Comité de Ética en Investigación de la Universidad Industrial de Santander, que atiende dicho hospital.

Los criterios de inclusión fueron: peso entre 750 y 1.500 g, edad de gestación entre 27 y 32 semanas, y que, al momento de ingreso al estudio, no hubiesen recibido ningún tipo de alimentación. Se excluían quienes tuvieran anomalías congénitas del sistema gastrointestinal, retardo del crecimiento intrauterino 0 que presentasen inestabilidad respiratoria o hemodinámica.

En estos niños se indagó el puntaje de Apgar al minuto y a los cinco minutos de nacimiento, la edad de gestación contada en semanas desde la fecha de la última menstruación materna, el tiempo transcurrido antes de su ingreso a las salas de recién nacidos y si habían sido sometidos a exanguinotransfusión o cateterismo umbilical.

Se calculó el tamaño de la muestra teniendo en cuenta que en las salas de recién nacidos se hospitalizan anualmente, en promedio, 90 niños prematuros con peso menor de $1.500 \mathrm{~g}$ de, de los cuales, $12 \%$ desarrolla enterocolitis necrosante. Se partió de la hipótesis de que los dos tipos de alimentación debían ser equivalentes y que la máxima diferencia esperada, en cuanto a la aparición de enterocolitis necrosante, no debía superar el $12 \%$. Se asumió una confiabilidad de $95 \%$ y poder de $80 \%$ para detectar las diferencias entre los grupos. Así, el tamaño de muestra por captar fue de 196 niños, 98 en cada uno de los tipos de alimentación.

La inclusión de los recién nacidos se hizo en forma consecutiva, hasta que se completó el tamaño esperado de la muestra. Los niños se asignaron al grupo de alimentación correspondiente mediante asignación aleatoria simple con una moneda, por una enfermera profesional, externa al equipo investigador. Se hicieron dos análisis interinos 
de resultados, cuando se completaron 100 y 200 pacientes ingresados.

Para el caso de los niños que habían quedado incluidos en el grupo de alimentación temprana, la vía oral se inició entre las 24 y las 48 horas de vida, siempre y cuando el paciente se encontrara en condiciones estables, no presentara distensión abdominal, no tuviese evidencia de sangrado gastrointestinal y hubiera actividad peristáltica (presencia de ruidos intestinales o paso de materia fecal). La alimentación entérica se inició con leche materna o fórmula láctea para prematuros, sin diluir, suministrando un mililitro cada seis horas. Al segundo día se inició el aumento en el volumen, así: tomas a intervalos de cada tres horas, con un volumen de un mililitro por toma, y al siguiente día con aumentos que no superaran los $20 \mathrm{ml} / \mathrm{kg}$ al día. Los incrementos en volumen continuaron de igual manera en los días subsiguientes, hasta llegar a $150 \mathrm{ml} / \mathrm{kg}$ diarios, siempre y cuando el paciente permaneciera estable y no presentara signos o síntomas de intolerancia a la vía oral.

En los neonatos en los que se inició la alimentación tardíamente -que era la práctica usual en la unidad neonatal- se tuvieron en cuenta los mismos criterios para el inicio de la vía oral temprana, pero con inicio al quinto día de vida. Igualmente, se les suministró leche materna o fórmula láctea para prematuros sin diluir, ofreciendo inicialmente un volumen correspondiente, en mililitros, a un tercio del $1 \%$ de su peso en gramos. El incremento se hizo de la misma forma que en el grupo de alimentación temprana.

La monitorización de la tolerancia a la vía oral se hizo teniendo en cuenta el residuo gástrico antes de administrar la siguiente toma. En caso de ser menor de $25 \%$, este residuo se retornaba y se continuaba con la siguiente toma sin modificaciones; si estaba entre 25 y $50 \%$, igualmente se retornaba y a la siguiente toma se le descontaba dicho residuo; si este residuo persistía por dos tomas, se disminuía el volumen a aquel que el paciente venía tolerando previamente. Finalmente, si el residuo era mayor de $50 \%$, se regresaba al volumen tolerado previamente sin darse la dosis correspondiente a esa toma. La vía oral se suspendía cuando el residuo tenía características achocolatadas, biliosas o con sangre.

Independientemente del grupo de alimentación al que hubieran sido asignados, a todos los pacientes se les inició alimentación parenteral a las 48 horas de vida y se continuó hasta que el niño pudiera recibir todos sus aportes calóricos por vía oral. Ningún menor recibió lactancia materna exclusiva o sólo formula láctea.

Diariamente, se midió el perímetro abdominal a nivel del ombligo y se evaluaron otros signos que permiten sospechar enterocolitis necrosante, como dolor a la palpación del abdomen, disminución de los ruidos intestinales o presencia de masa. En caso de diarrea, se midieron los azúcares reductores y se hizo la prueba de guayaco. Se descartó que la presencia de sangre se debiera a fisuras anales 0 a sangre materna deglutida.

Aparte de la causa mencionada anteriormente, también se suspendió la vía oral cuando se encontró aumento del perímetro abdominal de más de $2 \mathrm{~cm}$ con respecto a la medición del día anterior, defensa abdominal o presencia de sangrado gastrointestinal. En ese momento, se solicitaba una radiografía simple de abdomen en posición vertical, tanto antero-posterior como lateral, y se tomaban muestras de sangre para hemograma, proteína $C$ reactiva y velocidad de sedimentación globular; además, se tomaban cultivos de sangre y materia fecal. Paso seguido, se iniciaba manejo antibiótico de acuerdo con las recomendaciones de Bell y las modificaciones de Wals y Kliegman (21). A todos los pacientes se les hizo seguimiento radiológico y clínico hasta establecer un diagnóstico definitivo del grado de enterocolitis. La lectura de las placas de abdomen fue hecha por el médico pediatra tratante y un radiólogo o cirujano pediatra, todos desconociendo el momento de ínicio de la vía oral.

Si no se lograba confirmar el diagnóstico de enterocolitis necrosante, la alimentación por vía entérica se reiniciaba tres días después, con el esquema que el paciente tenía asignado.

El desenlace primario de este estudio fue el diagnóstico de enterocolitis necrosante, según los criterios de Bell modificados por Wash y Kliegman (21), en estadio IIA o superior. Como desenlaces secundarios se consideraron el tiempo de estancia hospitalaria medido en días, desde el ingreso hasta que el paciente era dado de alta, y el cambio promedio de peso entre estos mismos días.

El análisis de los datos se adelantó utilizando el paquete estadístico Stata 10.1® (StataCorp, College Station, TX, Estados Unidos). Para la evaluación de la hipótesis del estudio, se analizaron los resultados por intención de tratar. Para ello, se 
utilizaron las pruebas de ji al cuadrado, la prueba exacta de Fisher, la t de Student o de Mann-Whitney, según fuese el caso, a fin de evaluar las diferencias entre los grupos de alimentación, asumiendo una confiabilidad de $95 \%$. Igualmente, se estimó el riesgo relativo (RR) con su intervalo de confianza (IC) respecto a la incidencia de enterocolitis necrosante y de mortalidad, en ambos grupos.

\section{Resultados}

Se asignaron 239 pacientes a los grupos de tratamiento, 135 al de alimentación temprana y 104 al de alimentación tardía (figura 1). Aunque el tamaño de la muestra inicialmente calculado fue de 98 pacientes en cada grupo del estudio, la asignación aleatoria simple a cada uno de ellos hizo que su tamaño fuera desequilibrado, por lo que se continuó incluyendo pacientes hasta completar el tamaño mínimo del grupo que tenía menos pacientes.

En el cuadro 1 se mencionan las características de los pacientes al ingreso. No se encontraron diferencias estadísticamente significativas entre los grupos respecto a los factores conocidos de riesgo para enterocolitis necrosante.

En los dos análisis interinos programados, no se encontraron diferencias en la incidencia de enterocolitis necrosante entre los dos grupos ni en la mortalidad, por lo que se llevó el estudio hasta completar el tamaño de muestra calculado. Al terminar el estudio, se habían presentado 23 casos de enterocolitis necrosante, 14 (10,4\%) en el grupo de alimentación temprana ( $\mathrm{IC}_{95} \% 6,0$ $17,1)$ y $9(8,7 \%)\left(\mathrm{IC}_{95} \% 4,3-16,2\right)$ en el grupo de alimentación tardía ( $R R=1,22 ; \quad I C_{95} \%$ 0,49-3,20, $p=0,652)$. Cinco $(3,7 \%)$ niños fallecieron en el grupo de alimentación temprana $\left(\mathrm{IC}_{95} \% 1,4-8,9\right)$ y ocho $(7,7 \%)$ en el de alimentación tardía $\left(\mathrm{IC}_{95 \%} 3,6\right.$ $15,0)$, sin que estas diferencias fueran significativas $\left(R R=0,46 ; \quad I C_{95} \% 0,12-1,60 ; p=0,182\right)$, según se presenta en el cuadro 2.

El tiempo promedio de estancia hospitalaria también fue similar entre los dos grupos; para el de alimentación temprana fue de 27,4 días [desviación estándar $(\mathrm{DE}=14,9)$ y, para el de alimentación tardía, 30,1 días $(D E=18,0 ; p=0,201)$. Por otro lado, el peso promedio al egreso de los pacientes asignados al grupo de alimentación temprana fue de $1.614 \mathrm{~g}$ ( $\mathrm{DE}=205 \mathrm{~g}$ ), mientras que, en el grupo de alimentación tardía fue de $1.701 \mathrm{~g}$ (DE=261 g; $p=0,004)$. Sin embargo, el incremento diario de peso a partir del peso al inicio de la alimentación entre los neonatos sobrevivientes, fue similar: $13,5 \mathrm{~g} / \mathrm{kg}$ al día $\left(\mathrm{IC}_{95} \% 12,1-14,8\right)$ en el grupo de alimentación temprana y $20,7 \mathrm{~g} / \mathrm{kg}$ al día $\left(\mathrm{IC}_{95} \%\right.$ $9,8-31,5)$ en el de alimentación tardía $(p=0,138)$.

La meta de la alimentación entérica total, de 150 $\mathrm{ml} / \mathrm{kg}$ al día, se alcanzó al décimo día en el grupo

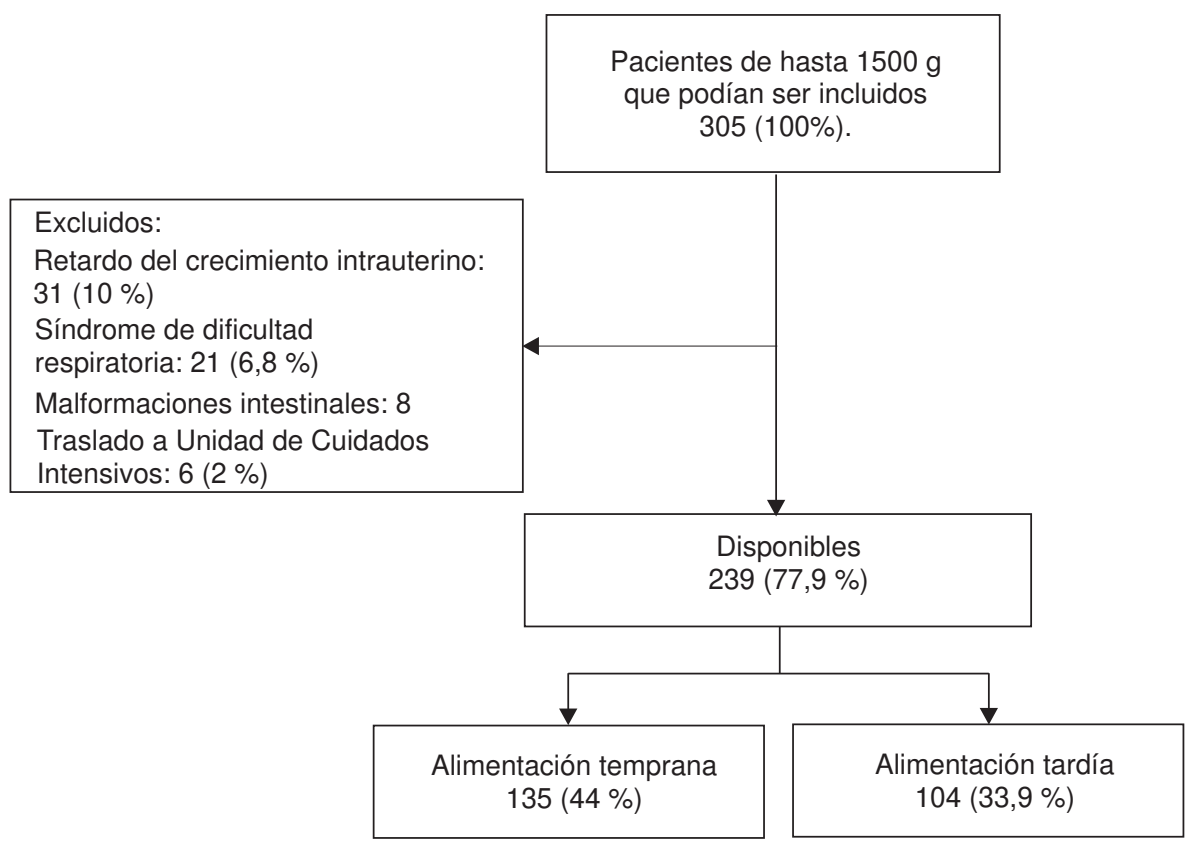

Figura 1. Selección de los participantes 
Cuadro 1. Características basales de los pacientes incluidos en el estudio

\begin{tabular}{|c|c|c|c|}
\hline Variables & $\begin{array}{l}\text { Alimentación temprana } \\
\qquad(n=135)(\%)\end{array}$ & $\begin{array}{l}\text { Alimentación tardía } \\
(n=104)(\%)\end{array}$ & $\mathbf{p}$ \\
\hline \multicolumn{4}{|l|}{ Demográficas y parto } \\
\hline Peso $(g)^{*}$ & $1.243(219)$ & $1.224(218)$ & 0,49 \\
\hline Edad de gestación (semanas)* & $30,3(2,0)$ & $30,0(2,1)$ & 0,25 \\
\hline Sexo masculino & $67(49,6)$ & $54(51,9)$ & 0,72 \\
\hline Al menos, un control prenatal & $83(61,5)$ & $63(60,6)$ & 0,89 \\
\hline Maduración pulmonar & $79(71,6)$ & $60(57,7)$ & 0,60 \\
\hline Cesárea & $96(71,6)$ & $75(72,1)$ & 0,93 \\
\hline $\begin{array}{l}\text { Reanimación con asistencia respiratoria } \\
\text { positiva }\end{array}$ & $39(28,9)$ & $41(39,4)$ & 0,09 \\
\hline Reanimación con intubación & $5(3,7)$ & $4(3,8)$ & 0,95 \\
\hline Apgar al minuto* & $6,9(1,39)$ & $6,7(1,4)$ & 0,24 \\
\hline Apgar a los cinco minutos* & $8,41(0,9)$ & $8,29(1,2)$ & 0,39 \\
\hline Número de partos maternos* & $2,3(2,0)$ & $2,66(2,3)$ & 0,19 \\
\hline \multicolumn{4}{|l|}{ Complicaciones obstétricas } \\
\hline Trastornos hipertensivos del embarazo & $23(17,0)$ & $27(25,9)$ & 0,09 \\
\hline Desprendimiento de placenta & $1(0,7)$ & $1(1,0 \%)$ & 0,85 \\
\hline Placenta previa & $2(1,5)$ & $4(3,8)$ & 0,45 \\
\hline Corioamnionitis & $22(16,3)$ & $12(8,9)$ & 0,29 \\
\hline \multicolumn{4}{|l|}{ Complicaciones del recién nacido } \\
\hline Sufrimiento fetal agudo & $16(11,8)$ & $14(13,5)$ & 0,71 \\
\hline Síndrome de dificultad respiratoria & $17(12,6)$ & $11(10,6)$ & 0,63 \\
\hline Presencia de infección & $82(60,7)$ & $57(54,8)$ & 0,36 \\
\hline Ictericia & $43(31,8)$ & $36(34,6)$ & 0,65 \\
\hline Cateterismo umbilical & $20(14,8)$ & $15(14,4)$ & 0,93 \\
\hline Policitemia & $2(1,5)$ & $3(2,9)$ & 0,45 \\
\hline Exanguinotransfusión & $1(0,7)$ & $1(1,0)$ & 0,85 \\
\hline
\end{tabular}

* Promedio y desviación estándar

Cuadro 2. Resultados en los grupos evaluados

\begin{tabular}{lccc}
\hline Desenlaces & $\begin{array}{c}\text { Alimentación temprana } \\
(\mathbf{n = 1 3 5 )}\end{array}$ & $\begin{array}{c}\text { Alimentación tardía } \\
(\mathbf{n = 1 0 4 )}\end{array}$ & $\mathbf{p}$ \\
\hline Incidencia de enterocolitis necrosante & $14(10,4 \%)$ & $9(8,7 \%)$ & 0,652 \\
Letalidad & $5(3,7 \%)$ & $8(7,7 \%)$ & 0,182 \\
Incremento diario de peso $(\mathrm{g} / \mathrm{kg}$ al día) & (13,5 (12,1 a 14,8) & $20,7(9,8$ a 31,5) & 0,138 \\
\hline
\end{tabular}

* Promedio e intervalo de confianza de $95 \%$

de alimentación temprana y, al noveno día, en el grupo de alimentación tardía.

\section{Discusión}

El presente estudio demuestra que no hay diferencias respecto a la frecuencia de enterocolitis necrosante si se inicia la vía oral en forma temprana o si se hace en forma tardía. El análisis post hoc del poder del estudio, dados los resultados del ensayo clínico, indican que éste es superior a $80 \%$ para hallar diferencias expresables con un riesgo relativo de 1,9 para la incidencia de enterocolitis necrosante, entre los dos grupos.

El uso de leche materna o de fórmula láctea para prematuros sin diluir es una novedad en este estudio, pues en la mayoría de los esquemas se recomienda iniciar con la fórmula diluida. Sin embargo, los resultados de este estudio adicionan más argumentos para afirmar que no hay razón para iniciar en forma tardía la alimentación entérica en los prematuros (21). Incluso, se ha encontrado que el inicio tardío de la vía oral aumenta el riesgo de desarrollar enterocolitis necrosante (22).

La enterocolitis necrosante aún no tiene una etiología definida. Entre los factores reconocidos como coadyuvantes en la presentación de la enfermedad, se encuentra la alimentación entérica (7). Sin embargo, se ha visto que la alimentación entérica mínima tiene ventajas en cuanto a que mejora la motilidad y la producción enzimática, y 
alcanza los aportes óptimos de manera más rápida, sin aumentar el riesgo de enterocolitis necrosante $(14-17,20)$.

Aún no hay un consenso sobre el papel de los aumentos del volumen de leche en la patogenia de la enterocolitis necrosante. En varios estudios se informa sobre esto $(20,23-25)$ y se señala que los incrementos de $20 \mathrm{ml} / \mathrm{kg}$ al día son seguros. En el presente estudio, se confirmó la seguridad de este incremento, en la medida en que la incidencia de enterocolitis en los dos grupos de pacientes fue similar, ambos dentro de valores razonables $(26,27)$. Es de resaltar que los pacientes incluidos en este estudio no se encontraban en situación de inestabilidad hemodinámica, de tal forma que los dos grupos eran uniformes en este aspecto. De la misma manera, la unificación de criterio diagnóstico utilizando la clasificación de Bell, dejó de lado la subjetividad y unificó la evaluación de los pacientes.

En el estudio de casos y controles de Henderson, et al. (28), se encontró que el tiempo de duración de la alimentación nutritiva o entérica mínima (menos de un mililitro por kilogramo de peso por hora) fue más corto en los casos (3,3 días) que en los controles (6,2 días) que corresponde a una diferencia media (DM) de 2,9 días ( $\mathrm{IC}_{95 \%}-4,9$ a-0,9). Los casos alcanzaron el volumen de alimentación completo más temprano que los controles: los casos, una media de 9,9 días $(\mathrm{DE}=4,2)$ después del nacimiento y, los controles, una media de 14,3 días $(\mathrm{DE}=9,8)\left(\mathrm{IC}_{95 \%}-7,3 \mathrm{a}-1,5\right)$. Esto sugiere que la duración de la alimentación nutritiva y el volumen de los incrementos de la alimentación pueden modificar el riesgo de presentar enterocolitis necrosante.

En el presente estudio, se suministró alimentación entérica mínima durante dos días. Berseth, et al. (29), hicieron un ensayo clínico en niños prematuros menores de 32 semanas; a un grupo, le suministraron $20 \mathrm{ml} / \mathrm{kg}$ de leche durante 10 días $y$, a otro, le hicieron incrementos diarios de $20 \mathrm{ml} /$ $\mathrm{kg}$ en el volumen del alimento y el desenlace fue enterocolitis necrosante. El estudio se suspendió tempranamente debido a que en el grupo con incremento diario del volumen del alimento, se presentaron siete casos de enterocolitis necrosante (10\%), porcentaje similar al del grupo de alimentación temprana en el presente estudio (10,4\%), en el cual también se incrementaron 20 $\mathrm{ml} / \mathrm{kg}$ al día, en comparación con el observado $(1,4$ $\%)$ en el grupo sin incremento de volumen. Esto hace pensar que prolongar los días en que se suministra alimentación entérica mínima, disminuye la incidencia de enterocolitis necrosante.

Se tenía el concepto de que entre más tarde se iniciara la alimentación entérica menos posibilidades había de enterocolitis necrosante. Sólo cuando empezaron a hacerse los estudios con esquemas de inicio temprano de alimentación entérica hipocalórica, se pudo demostrar que estos esquemas no aumentaban el riesgo de enterocolitis necrosante y sí mejoraban la producción enzimática y hormonal, y la motilidad intestinal $(14,17)$.

En este estudio, los pacientes recibieron alimentación entérica mínima o nutritiva sólo por dos días, en contraposición a los esquemas que recomiendan prolongar más días estos pequeños aportes de nutrientes. El estudio de Berseth, que se suspendió tempranamente al encontrarse un aumento inusitado de casos de enterocolitis necrosante en el grupo que no tuvo alimentación entérica mínima, implica que son necesarios nuevos ensayos clínicos para comparar los esquemas de alimentación con diferentes días de alimentación entérica mínima o nutritiva, para definir el tiempo óptimo de su duración.

Una debilidad del presente estudio es que no se hizo una unificación previa de la lectura de las imágenes radiológicas entre los cirujanos pediatras y los radiólogos. Sin embargo, no se presentaron diferencias en la frecuencia de enterocolitis necrosante grave, cuyo cuadro clínico no se prestaba para confusión en la interpreción. El método de asignación aleatoria simple con moneda es otra debilidad, aunque al comparar los grupos con respecto a los factores de riesgo conocidos, no se encontró diferencia alguna.

Los actuales resultados reafirman aún más que la práctica de iniciar en forma temprana la alimentación no aumenta el riesgo de enterocolitis necrosante y sí representa grandes ventajas. Esto incluye la práctica de no diluir la leche y el uso de un período muy corto de alimentación entérica hipocalórica de tan sólo dos días. La estandarización del proceso de atención de los niños prematuros en cuanto al método de alimentación, disminuye la incidencia de enterocolitis necrosante, tal como lo demostraron Tyson y Kennedy en un metanálisis de estudios observacionales $(18,19)$. La explicación está relacionada con que la estandarización del proceso elimina de forma importante la yatrogenia, evitando que en algunos pacientes se inicien esquemas inconvenientes de alimentación. 


\section{Conflicto de interés}

Los autores no tienen conflictos de interés que declarar.

\section{Financiación}

El estudio fue realizado como parte de la atención regular que los pacientes prematuros reciben en el servicio de recién nacidos donde se adelantó el estudio y no recibió patrocinio por entidad alguna.

\section{Referencias}

1. Neu J. Necrotizing enterocolitis: The search for a unifying pathogenic theory leading to prevention. Pediatr Clin North Am. 1996;43:409-32.

2. Hsuell W, Caplan MS, Qu XW, Tan XD, Depluen IG, Gonzalez-Crussi F. Neonatal necrotizing enterocolitis: Clinical considerations and pathogenic concepts. Pediatr Dev Pathol. 2003;6:6-23.

3. Covert RF, Neu J, Elliott MJ, Rea JL, Gimotty PA. Factors associated with age of onset of necrotizing enterocolitis. Am J Perinatol. 1989;6:455-60.

4. Stoll BJ. Epidemiology of necrotizing enterocolitis. Clin Perinatol. 1994;21:205-18.

5. Llanos AR, Moss ME, Pinzón MC, Dye T, Sinkin RA, Kendig JW. Epidemiology of neonatal necrotizing enterocolitis; a population-based study. Paeditr Perinat Epidemiol. 2002;16:342-9.

6. Doring J, Kempley S, Leaf A. Feeding growth restricted preterm infants with abnormal antenatal Doppler results. Arch Dis Child Fetal Neonatal Ed. 2005;90:359-63.

7. Anderson DM, Kliegman RM. The relationship of neonatal alimentation practices to the occurrence of endemic necrotizing enterocolitis. Am J Perinatol. 1991;8:62-7.

8. Berseth CJ. Feeding strategies and necrotizing enterocolitis. Curr Opin Pediatr. 2005;17:170-3.

9. Martin CR, Walker WA. Intestinal immune defenses and the inflammatory response in necrotizing enterocolitis. Sem Fetal Neonatal Med. 2006;11:e369-77.

10. Neu J. The "myth" of asphyxia and hypoxia-ischemia as primary causes of necrotizing enterocolitis. Biol Neonate. 2005;87:97-8

11. Lin PW, Narsr TR, Stoll BS. Necrotizing enterocolitis: Recent scientific advances in pathophysiology and prevention. Semin Perinatol. 2008:32;70-82.

12. Spritzer R, Koolen AM, Baerts W, Fetter WP, Lafeber HN, Sauer PJ. A prolonged decline in the incidence of necrotizing enterocolitis after the introduction of a cautions feeding regimen. Acta Paediatr Scand. 1988;77:909-11.

13. Brown EG, Sweet AY. Preventing necrotizing enterocolitis in neonates. JAMA. 1978;240:2452-4.

14. Berseth CL. Effect of early feeding on maturation of the preterm infant's small intestine. J Pediatr. 1992;120:947-53.
15. Berseth CL, Nordyke C. Enteral nutrients promote postnatal maturation of intestinal motor activity in preterm infants. Am J Physiol. 1993;264:G1046-51.

16. McClure RJ, Newell SJ. Randomized controlled study of clinical outcome following trophic feeding. Arch Dis Child Fetal Neonatal Ed. 2000;82:F29-33.

17. Tyson JE, Kennedy KA. Minimal enteral nutrition in parenterally fed neonates. Cochrane Database Syst Rev. 2000;(2):CD000504. Update in: Cochrane Database Syst Rev. 2005;(3):CD000504

18. Kennedy KA, Tyson JE, Chamnanvanakij S. Rapid versus slow rate of advancement of feedings for promoting growth and preventing necrotizing enterocolitis in parenterally fed low-birth-weight infants. Cochrane Database Syst Rev. 2000;(2):CD001241. Update in: Cochrane Database Syst Rev. 2008;(2):CD001241.

19. Book LS, Herbst JJ, Jung AL. Comparison of fast and slow feeding rate schedules to the development of necrotizing enterocolitis. J Pediatr. 1976;89:463-6.

20. Rayyis SF, Ambalavanan N, Wright L, Carlo WA. Randomized trial of "slow" versus "fast" feed advancements on the incidence of necrotizing enterocolitis in very low birth weight infants. J Pediatr. 1999;34:293-7.

21. Walsh MC, Kliegman RM. Necrotizing enterocolitis: Treatment based on staging criteria. Pediatr Clin North Am. 1986;33:179-201.

22. LaGamma EF, Ostertag SG, Birenbaum H. Failure of delayed oral feedings to prevent necrotizing enterocolitis. Results of study in very-low-birth-weight neonates. Am J Dis Child. 1985;139:385-9.

23. Okada Y, Klein N, Pierro A. Small volumes of enteral feedings normalize immune function in infants receiving parenteral nutrition. J Pediatr Surg. 1998;33:16-9.

24. Book LS, Herbsf JJ, Jung AL. Comparison of fast and slow feeding rate schedules to the development of necrotizing enterocolitis. J Pediatr. 1976;89:463-6.

25. Caple J, Armentrout D, Huseby V, Halbardier B, García J, Sparks JW, et al. Randomized controlled trial of slow versus rapid feeding volume advancement in preterm infants. J Pediatr. 2004;114:1597-600.

26. Henderson G, Craig S, Brocklehurst P, McGuire W. Enteral feeding regimens and necrotizing enterocolitis in preterm infants: Multicentre case-control study. Arch Dis Child Fetal Neonatal Ed. 2007;94:120-3.

27. Patole SK, de Klerk N. Impact of standardized feeding regimens on incidence of neonatal necrotizing enterocolitis: A systematic review and meta-analysis of observational studies. Arch Dis Child Fetal Neonatal Ed. 2005;90:F147-51.

28. Henderson G, Craig S, Brucklehurst P, McGuire W. Enteral feeding regimens and necrotizing enterocolitis in preterm infants: A multicenter case-control study. Arch Dis Child Fetal Neonatal Ed. 2009;94:F120-3

29. Berseth CL, Bisquera JA, Pafe VU. Prolonging small feeding volumes early in life decreases the incidence of necrotizing enterocolitis in very low birth weight infants. Pediatrics. 2003;11:529-34. 